\title{
MIRROR-IMAGE STIMULATION APPLIED TO FIELD BEHAVIORAL STUDIES ${ }^{1}$
}

\author{
Gerald E. Svendsen and Kenneth B. Armitage \\ Division of Biological Sciences, University of Kansas, Lawrence 66044
}

\begin{abstract}
Twenty-nine adult and yearling marmots were exposed to mirror-image stimulation. Marmots interacted intensely with their image, and the frequency of occurrences of 22 behaviors was recorded. The four factors extracted from the behavioral data by factor analysis accounted for $88 \%$ of the total variance. Three factors were interpretable and designated "approach," "avoidance," and "sociability." Behavioral patterns were stable over time and repeatable. A plot of "avoidance" against "sociability" provides a visualization of the numerical behavioral profile of each animal which is consistent with and interpretable in terms of behavioral patterns observed in the field.
\end{abstract}

\section{INTRODUCTION}

Mirror-image stimulation (MIS) is a technique for studying aggressive and social patterns in a wide variety of animals which respond to their reflection in a mirror (Gallup 1968). Male Siamese fighting fish (Betta splendens), parakeets (Melopsittacus undulatus) and finches (Passer domesticus) prefer interaction with their image in MIS over visual access to another conspecific (Baenninger 1966, Gallup and Capper 1970). MIS seems similar to visual confrontation with another animal of the same species, but the stimulus characteristics of an animal's reflection are different from visual stimuli of another animal (Gallup and Capper 1970). Advantages of using MIS over direct confrontation with another animal in studying agonistic behavior include elimination of all except visual stimuli and absence of physical conflict.

The theory that social behavior regulates population density was developed by Wynne-Edwards (1962), and numerous workers have suggested the importance of behavioral interactions to population regulation in small animals (Chitty 1953, Southwick 1955, Bronson 1964, Krebs 1970, Downhower and Armitage 1971, Meyers and Krebs 1971). Intraspecific behavior is often ascertained from observations on social interactions in the field (Armitage 1962, 1965 ) or from forced paired encounters in a neutral arena (Bronson 1964, Krebs 1970). Both methods lack experimental control over the stimulus situation, and the latter technique allows for direct physical confrontation between combatants that may result in physical damage to the participants. Krebs (1970) also uses a measure of open-field exploratory behavior in addition to aggressive behavior to arrive at behavior changes in voles, Microtus ochrogaster and $M$. pennsylvanicus.

The present study presents a technique using factor analysis of behavioral patterns of individual yellowbellied marmots (Marmota flaviventris) observed

${ }^{1}$ Received April 6. 1972; accepted October 6, 1972. during MIS to derive numerical behavior profiles which then are applied to the interpretation of behavior observed in the field.

\section{Methods}

During the summer of 1971 , we trapped with National Live Traps 29 yearling and adult yellow-bellied marmots in the vicinity of the Rocky Mountain Biological Laboratory, Colorado, and exposed them to MIS. The apparatus consisted of a square arena 92 $\mathrm{cm} \times 92 \mathrm{~cm}$ and $31 \mathrm{~cm}$ high. The top and bottom of the arena were covered with hardware cloth of $6 \mathrm{~mm}$ mesh, and the sides were pine boards. The entrance was through the back, opposite a wall having a glass mirror about $43 \mathrm{~cm} \times 30 \mathrm{~cm}$, covered with a removable opaque partition, which could be lifted to expose the mirror. Food consisting mainly of freshpicked dandelion (Taraxacum) and cow parsnip (Heracleum) was placed along one side of the arena at midcage.

Marmots were exposed between $08.00-11.00$ hours and 16.00-19.00 hours, periods corresponding to the natural activity cycle of the animals (Armitage 1962). Animals captured late in the morning or evening were held in live-traps at the laboratory until the next test period. In order to mask extraneous noise, we located the experimental appartus in an isolated willow thicket next to a stream. We observed from a blind atop a $1.5-\mathrm{m}$ tower that allowed us to view the entire arena and clearly observe the marmots' behavior.

Individual marmots were released into the arena and permitted to explore and acclimate to the situation for 15 minutes before the partition was removed and MIS begun. The geometry of the arena was such that a marmot could avoid seeing itself only by facing directly into one of the four corners or directly away from the mirror at the back. The partition was removed when the animal was facing the position of the mirror, insuring that an initial stimulus would occur. The activities of each marmot were recorded 
TABle 1. Twenty-two behavioral categories recorded for each animal during mirror image stimulation

\section{1. chirping}

2. sitting

3. lying

4. eating

5. arena investigation

6. nose contact with image

7. pawing and muzzling of image

8. lying with head flat to ground, oriented to image

9. lunging at image

10. tooth chatter

11. tail wagging

12. head bobbing or weaving

13. conflict behavior ${ }^{\mathrm{a}}$

14. freezing when seeing image

15. retreating from image

16. activities occurring under $30 \mathrm{~cm}$ from mirror

17. activities occurring in front half of arena

18. activities occurring in back half of arena

19. orientation towards or parallel to image

20. orientation away from image or no orientation

21. time to approach ${ }^{b}$

22. self-grooming

aConflict behavior is used here to include a unique behavior consisting of many shehavior is used here to include a unique behavior consisting rapid withdrawals, not resulting in total approach to the mirror.

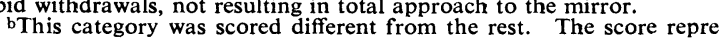
ented the time during the 15 -minute exposure that the animal approached its image and made contact. If an animal approached in the 2 nd minute the score was 2 . A score of 16 was given to those that never approached the mirror.

in 1-minute intervals over a 15-minute test period. After the test the mirror was washed. Although there was no way to remove possible odor from the arena after each run, we found no difference in the distribution of scores from individuals run after an aggressive animal and those following a social one $(P<.01$, Mann-Whitney $U$ test; Siegel 1956, p. 116), indicating that odor was not a stimulus in the test. Marmots were then released where they were trapped. During the 11-year study, including trapping, marking, removal of animals for surgical implantations, and MIS, we have never observed any change in social behavior of a marmot due to our procedures.

The behavioral data were analyzed by factor analysis. Although canonical, multiple, and partial correlation analysis, discriminant functions, and principal components also are statistical methods available to treat multivariate data (Cooley and Lohnes 1971, p. 96), we chose factor analysis because it is a technique for reducing a large number of correlated phenomena into a small number of uncorrelated variables, because a large number of phenomena can be handled with the assistance of a computer, and because the technique can be used to handle many different kinds of data over a wide range of research designs (Rummel 1970, p. 3).

Behavioral data were arranged into 22 categories (Table 1). A category was scored if the behavior it represents occurred once or more during a 1-minute interval. If a categorized behavior occurred in all fifteen 1-minute intervals, the category received the maximum score of 15 . Individual scores form a vector; therefore each run determines a vector of 22 elements, each element ranging from zero to 15 . The data matrix consists of 33 runs ( 29 individuals with 4 replicate runs) as rows and 22 behavioral categories as columns. A matrix of product-moment correlation coefficients $(33 \times 33)$ was computed on the data matrix.

Eigenvalues were extracted from the correlation matrix, and only eigenvalues above 1.0 were considered in determining the factors. Factors were reextracted until the communality estimates stabilized and the factor matrix was then rotated to simple structure using Kaiser's Normal Varimax Method (Rummel 1970).

In this technique we restricted our analysis to responses during the exposure period. However, we realize that additional information could be attained by analyzing the behavior during the pre-exposure and post-exposure periods.

\section{Results}

The immediate response observed in all marmots when exposed to MIS is to stop whatever they are doing and to direct their attention to the image. Some animals freeze in a particular stance for several minutes, whereas others assume subsequent activities after only a momentary hesitation. A marmot may either approach the mirror or retreat to the back of the arena after the initial hesitation. Those that approach the mirror may do so cautiously and hesitate often during their approach or they may approach with no apparent hesitation. Vocalizations are common during MIS. Some animals that are vocal during pretest become silent during MIS; others are silent during acclimation and become vocal during MIS.

Investigation of the arena occurs during the acclimation period as well as the test period. Again, some of the animals which exhibit restraint during the acclimation become very active after exposure to their image, whereas others become inactive after seeing their reflection. If a marmot approaches its image, it may exhibit positive social actions, such as nose contact and pawing and muzzling of the image, or it may exhibit aggressive activities, such as tooth chatter and lunges at the mirror. Extremely social animals spend much of their time in the front half of the arena near the mirror engaged in apparent attempts to contact their image. Some, after a few minutes of fruitless attempts to make contact, simply sit or lie down next to the mirror. Aggressive animals also spend most of the exposure period near the mirror and oriented towards the image, but engaged in nonsocial activities.

Marmots that avoid contact with their reflections stay still during almost all the test period at a point farthest from the mirror, but oriented towards or 
TABLE 2. Varimax rotated factor matrix of 29 marmots

\begin{tabular}{|c|c|c|c|c|c|c|c|}
\hline Animals & $\operatorname{sex}^{b}$ & $\begin{array}{c}\text { age } \\
\text { class }^{\mathrm{c}}\end{array}$ & $\begin{array}{l}\text { date } \\
\text { run }\end{array}$ & $I^{d}$ & II & III & IV \\
\hline $1 a$ & $\mathbf{F}$ & A & 27 June & 0.80 & 0.29 & & \\
\hline $1 b$ & & & 12 July & 0.85 & 0.27 & & \\
\hline lc & & & 11 Aug. & 0.83 & & 0.38 & \\
\hline 2 & $\mathbf{M}$ & $\mathbf{Y}$ & 29 June & 0.85 & & & \\
\hline 3 & $\mathbf{F}$ & $\mathbf{A}$ & 10 July & 0.92 & & & \\
\hline 4 & $\mathbf{F}$ & $\mathbf{A}$ & 14 July & 0.82 & & & 0.50 \\
\hline 5 & $\mathbf{F}$ & $\mathbf{Y}$ & 8 July & 0.95 & & & \\
\hline 6 & 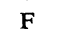 & $\mathbf{Y}$ & 14 July & 0.90 & & & \\
\hline 7 & $\mathbf{F}$ & $\mathbf{A}$ & 3 Aug. & 0.80 & 0.26 & 0.27 & \\
\hline 8 & $\mathbf{F}$ & A & 31 July & 0.89 & & 0.38 & \\
\hline 9 & $\mathbf{M}$ & $\mathbf{A}$ & 11 July & 0.86 & & 0.46 & \\
\hline 10 & $\mathbf{F}$ & $\mathbf{A}$ & 14 July & 0.58 & & 0.37 & 0.70 \\
\hline 11 & $\mathbf{M}$ & $\mathbf{A}$ & 9 July & 0.81 & & 0.48 & \\
\hline 12 & $\mathrm{~F}$ & A & 7 Aug. & 0.62 & & 0.67 & \\
\hline $13 a$ & $\mathbf{F}$ & $\mathbf{Y}$ & 14 July & 0.75 & & 0.61 & \\
\hline $13 b$ & & & 29 June & 0.34 & & 0.83 & \\
\hline 14 & F & $\mathbf{A}$ & 8 July & 0.57 & & 0.78 & \\
\hline 15 & $\mathbf{F}$ & $\mathbf{A}$ & 7 July & 0.32 & & 0.76 & \\
\hline 16 & $\mathbf{M}$ & A & 15 July & 0.32 & & 0.85 & \\
\hline 17 & $\mathbf{M}$ & A & 1 July & 0.34 & & 0.89 & \\
\hline 18 & $\mathbf{F}$ & A & 30 July & & & 0.81 & \\
\hline 19 & $\mathbf{F}$ & A & 16 July & & 0.32 & 0.82 & \\
\hline 20 & $\mathbf{F}$ & $Y$ & 1 July & & & 0.93 & \\
\hline 21 & $\mathbf{M}$ & A & 18 July & & 0.70 & & \\
\hline 22 & $\mathbf{F}$ & A & 15 July & & 0.73 & & \\
\hline 23 & $\mathrm{~F}$ & A & 27 June & 0.26 & 0.87 & & 0.27 \\
\hline 24 & $\mathbf{F}$ & $\mathrm{Y}$ & 5 July & & 0.89 & & 0.32 \\
\hline 25 & $\mathbf{M}$ & A & 1 July & & 0.98 & & \\
\hline 26 & $\mathbf{F}$ & $\mathbf{A}$ & 15 July & & 0.98 & & \\
\hline $27 \mathrm{a}$ & $\mathbf{M}$ & $\mathbf{Y}$ & 7 July & & 0.97 & & \\
\hline $27 b$ & & & 3 Aug. & & 0.97 & & \\
\hline 28 & $\mathrm{~F}$ & $\mathbf{Y}$ & 5 July & & 0.97 & & \\
\hline 29 & $\mathbf{M}$ & $\mathbf{A}$ & 16 June & & 0.97 & & \\
\hline \multicolumn{4}{|c|}{ Percent variance explained } & $38 \%$ & $30 \%$ & $26 \%$ & $6 \%$ \\
\hline
\end{tabular}

aLower case letters after the same number (e.g. la, lb, lc) represent replicate runs of the same individual.

${ }^{b} \mathbf{F}=$ female; $\mathbf{M}=$ male.

$\mathrm{C} A=$ adult $; \mathrm{Y}=$ yearling
$\mathrm{d}$ Zero factor loading is 0.2500 throughout

parallel to it. Frequently, vocalizations (10-15 chirps per minute) accompany this behavior. Some animals begin to approach after 10-12 minutes exposure but few of these marmots make contact with their image during the test period. One individual cowered in a farthest corner for the entire period.

Deviation from a normal distribution of activity as measured by the frequency of occurrence of an animal in the front half of the arena during the 15minute test, is significant $(P<0.01)$, KolmogorovSmirnov test, Siegel 1956, p. 47). The distribution of activity is bimodal, skewed towards occurrence in the front and in the back of the arena.

\section{Analysis of data}

Twenty-nine marmots were included in the analysis. Four factors were extracted from the behavioral data, accounting for $88 \%$ of the total variance (Table 2 ). Factors were interpreted by comparing the behavior of marmots grouped together on each factor and then classifying each factor according to the overall and unifying characteristic behavior of the marmots in that group.

The factor matrix of 29 marmots (Table 2) can be interpreted as follows: Factor I "approach" is represented by marmots who spend most of their time in the front half of the arena, sitting or lying near the mirror, oriented towards or parallel to their image. This factor does not differentiate the socially positive animals from the aggressive, so antisocial behaviors such as head bobbing or weaving, lying with the head flat on the ground, and lunging at the mirror, and tooth chatter are included. This factor explains $38 \%$ of the total variation.

Factor II is designated "avoidance" because animals in this group sit or lie at the back of the arena, oriented towards or parallel to their image, and do not approach the mirror or only do so hesitantly. This factor explains $30 \%$ of the total variation. Factor III is an axis containing marmots engaged in non-aggressive activities and will be called "sociability." Animals with greatest loadings in this group frequent the front half of the area, make much contact with the mirror, investigate the arena, eat, and engage in activities where they are not oriented to their image. This factor accounts for $26 \%$ of the total variation. Factor IV explains only $6 \%$ of the total variation and can not be interpreted biologically. A plot of factor II on factor III (Fig. 1) gives the best visual and biologically interpretable representation of the data.

\section{DISCUSSION}

The intense interaction of marmots with their image in the absence of both auditory and olfactory cues associated with the image is consistent with findings using other animals (Gallup and Capper 1970). MIS provides a technique for studying intra-

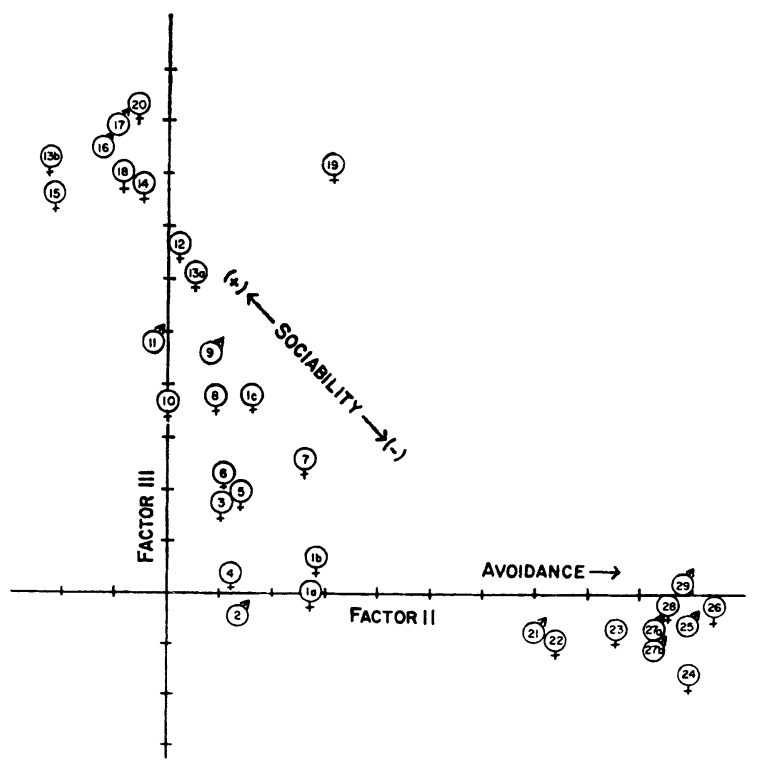

FIG. 1. Twenty-nine marmots plotted in the space of orthogonal factors II and III. Encircled symbols are the same as those in Table 2, designating individual animals and replicate runs. Sex is also indicated. 
specific interactions in marmots with greater control over the experimental conditions than the techniques commonly used. During MIS the stimulus modality remains constant and the animal completely controls the image; the reflected image cannot respond independently and so is completely compatible with the behavior of the animal. The image can never present a submissive or aggressive gesture unless it is initiated by the interacting animal.

The intensity of interaction by marmots during MIS is striking and each animal responds in its own way within the general reponse patterns. The intensity and uniqueness of the interaction may be because an animal's mirror reflection appears twice as far away as the animal is from the mirror. When an animal approaches the mirror perpendicularly, the apparent approach speed and distance of a position change is twice that of the animal's actual movement; and when an interacting marmot advances toward the mirror, both animal and image seem to be approaching a common midpoint. The reflected animal is as active as the interacting animal. The stimulatory effect of MIS therefore may closely approximate a natural encounter and elicit a close approximation of the behavior expressed during social encounters in the field. When quantified, this objective behavioral profile of each marmot may be applied to other studies in the biology of this species.

The responses of marmots to their reflected image are consistent with the behavior displays noted during social interactions in the field (Armitage 1962, 1965, authors' observations). Elements of play, greeting, grooming, vocalization, and agonistic behavior are expressed during MIS.

The behavior profiles of three marmots exposed to MIS more than once during the summer shows that this technique gives repeatable results over time (Fig. 1, see animals 1, 13, and 27). Adult female 1 tested aggressive all three times over a 3-month period. The first two exposures $(1 \mathrm{a}, 1 \mathrm{~b})$ provided very aggressive profiles and the third (1c) less so (Fig. 1). This decrease in aggressiveness is consistent with the conclusion of Armitage (1965) that agonistic behavior is greatest during reproduction and declines as marmots approach hibernation. Yearling female 13 displayed a high degree of sociability (13a, $13 \mathrm{~b})$ and yearling male 27 exhibited avoidance (27a, $27 b$ ) in each test.

The repeatability of specific responses from the same marmot during subsequent MIS is also striking. During "avoidance," adult male 21 expressed an unusually high amount of conflict behavior, consisting of many short approaches varying from one to five steps, followed by rapid withdrawals, never resulting in total approach to the mirror. After the initial 15minute test, the mirror was covered and the animal was allowed to return to other activities (i.e., explo- ration of the arena and eating), which he did vigorously. The mirror was uncovered again and the same conflict pattern was repeated.

\section{Test performance versus behavior in the field}

Many yellow-bellied marmots live in harems consisting of one territorial male to many females, yearlings, and young. All male yearlings are driven from the group and many yearling females also disperse. A social hierarchy exists among the harem females, and the degree of aggressiveness of the most aggresive female limits recruitment into the colony (Downhower and Armitage 1971).

The behavioral profiles derived from factor analysis of MIS (Fig. 1) fit well with our field observations of social behavior and activity patterns. Of those animals testing as minimal "sociability" and maximal "avoidance," 21, 23, and 29 lived as isolates. Male 25 appeared briefly at a colony, lived alone for a time on the periphery, then disappeared. Three yearlings, 24, 27, and 28, shared a burrow system with their mother at the north edge of the colony. They avoided contact with other adults; the male yearling fled whenever the resident male came near. The yearlings frequently "greeted" or groomed their mother. These socialization contacts were nearly always initiated by the yearlings. Although yearlings generally wander extensively over the area occupied by the colony, these yearlings limited their excursions to areas not utilized by other members of the colony. Females 22 and 26 were 3 -year-old sibs who avoided other adults. Avoidance was expressed by "alert" behavior or fleeing at the approach of another adult and by unstable home ranges. That is, these animals frequently shifted home burrow sites and feeding areas and generally wandered over a greater area of the colony habitat than do resident females. Socialization behaviors were few and usually were with a resident male. By contrast, a third sib, 14, who tested as highly social (Fig. 1), had a stable home range, did not avoid other animals, and had frequent socialization behavior with older adults.

Animals testing as minimal "sociability" and minimal "avoidance" also tested as aggressive. Females 1 and 7 lived alone in a habitat which appeared to be minimal for support. Contact with other nearby adults was never observed, but must have occurred with males as both had a litter of young. Female 10 lived with a male in what appeared to be limited habitat. The yearling male 2 was clearly subordinate to adults, but initiated many wrestling bouts with other yearlings and grappled with adult females. Contrary to the typical behavior of male yearlings, he remained in the colony of his birth throughout most of his second summer of life. Females 5 and 6 also were yearlings who remained in their colony area despite occasional agonistic encounters with adults. Their 
behavior profiles and field behavior support the hypothesis of Downhower (1968) that aggressive female yearlings may establish themselves in a colony in the presence of agonistic adult females. In one colony, the aggressive females were 3 and 4 . Each female excluded all other animals (except their young and adult males) from their home ranges. Yearling 6 occupied a neutral zone between the areas utilized by 3 and 4 . Yearling 5, however, occupied a prime home burrow site in her colony area near burrows inhabited by females 14 and 19 . Yearling 5 rarely had behavioral interactions with these females because she frequented feeding areas not utilized by the adults. Animals 9 and 11 were resident, territorial males; 8 was a resident female who moved into a vacant colony site.

Those animals testing as maximal "sociability" are most difficult to interpret. Marmots 16 and 17 were territorial males; 16 was in the process of establishing territorial rights, although subordinant to an adjacent male, whereas 17 was solitary but was seen to attackand drive off a smaller animal. Females 12, 14, 15, 18, and 19 were members of harems (Downhowe: and Armitage 1971). Although their behavioral characteristics varied, none were highly aggressive toward other females living in the same harems. Perhaps these animals may best be characterized as mildl! $\rightarrow$ aggressive or "socially confident." That is, they did not attempt to obtain exclusive use of an area, but were not intimidated by other animals. The remaining animals, 13 and 20, were yearling females. Both were sibs of female 6. Although yearling 13 wa $\rightarrow$ highly social, her behavior paralleled that characterized as submissive in field observations. She dispersed from her colony of birth and was seen briefly on the periphery of another colony about $350 \mathrm{~m}$ distant. Yearling 20 was closely associated with year $\rightarrow$ lings 2 and 6 . All three frequently wrestled with one another and were closely associated until late summer. At this time high vegetation and loss of mark ings because of molting made positive identification impossible and we are uncertain which animals emigrated and which were present.

This brief description of the relationship of the behavioral profiles derived from MIS to field observations was not mean to be a detailed analysis. Considerably more field data are available, but only gen $\rightarrow$ eral relationships were presented here in order to demonstrate the usefulness of MIS for field studies. Most interesting is that the continuum of profiles resulting from the analysis of MIS is consistent with the expected biological variation of animals and with the high degree of variability observed in the field.

\section{ACKNOWLEDGMENTS}

This study was made possible by Grant GB-8526 from the National Science Foundation and by a grant from the Margaret $\mathrm{H}$. and James E. Kelley Foundation, Inc. Facilities were provided by the Rocky Mountain Biological Laboratory, Inc. The authors wish to thank Dr. Rudolph Jander, National Science Foundation Senior Foreign Scientist, for his critical review of the manuscript and valuable suggestions.

\section{Literature Cited}

Armitage, K. B. 1962. Social behaviour of a colony of the yellow-bellied marmot (Marmota flaviventris). Anim. Behav. 10: 319-331.

$\rightarrow-$. 1965. Vernal behaviour of the yellow-bellied marmot (Marmota flaviventris). Anim. Behav. 13: 59-68.

Baenninger, R. 1966. Waning of an aggressive motivation in Betta splendens. Psychol. Sci. 4: 241-242.

Bronson, F. H. 1964. Agonistic behavior in woodchucks. Anim. Behav. 12: 470-478.

Chitty, D. 1952. Mortality among voles (Microtus agrestis) at Lake Vyrnwy, Montgomeryshire in 1936-9. Philos. Trans. R. Soc. Lond., Ser. B, 236: 505-552.

Cooley, W. W., and P. R. Lohnes. 1971. Multivariate data analysis. John Wiley and Sons, New York.

Downhower, J. F., and K. B. Armitage. 1971. The yellow-bellied marmot and the evolution of polygamy. Am. Nat. 105: 355-370.

Downhower, J. F. 1968. Factors affecting the dispersal of yearling yellow-bellied marmots (Marmota flaviventris). Ph.D. Thesis. Univ. Kansas, Lawrence.

Gallup, G. G., Jr. 1968. Mirror-image stimulation. Psychol. Bull. 70: 782-793.

Gallup, G. G., Jr., and S. A. Capper. 1970. Preference for mirror-image stimulation in finches (Passer domesticus domesticus) and parakeets (Melopsittacus undulatus). Anim. Behav. 18: 621-624.

Krebs, C. J. 1970. Microtus population biology: Behavioral changes associated with the population cycle in $M$. ochrogaster and $M$. pennsylvanicus. Ecology 51: 34-52.

Meyers, J. H., and C. J. Krebs. 1971. Genetic, behavioral, and reproductive attributes of dispersing field voles Microtus pennsylvanicus and $M$. ochrogaster. Ecol. Monogr. 41: 53-78.

Rummel, R. J. 1970. Applied factor analysis. Northwestern Univ. Press, Evanston, Illinois.

Siegel, S. 1956. Nonparametric statistics for the behavioral sciences. McGraw-Hill Book Co., New York.

$\rightarrow$ Southwick, C. H. 1955. Regulatory mechanisms of house mouse populations: Social behavior affecting litter survival. Ecology 36: 627-634.

Wynne-Edwards, V. C. 1962. Animal dispersion in relation to social behavior. Oliver and Boyd, Edinburgh. 